\title{
CONDUCTIVIDAD TÉRMICA DE COMPUESTOS TIPO SÁNDWICH USADOS EN LA INDUSTRIA DE LA CONSTRUCCIÓN
}

\section{THERMAL CONDUCTIVITY OF SANDWICH TYPE COMPOUNDS USED IN THE CONSTRUCTION INDUSTRY}

\author{
Marco Mendoza-Reyes ${ }^{1}$ (D), Mc Joshua Miguel Piñas-Moya' ${ }^{1}$ (i) \\ Manfred Horn-Mutschler1미, Mónica Gómez-León ${ }^{1}[1$
}

${ }^{1}$ Facultad de Ciencias, Universidad Nacional de Ingeniería, Lima, Perú

Recibido (Received): 23/03/2021 Aceptado (Accepted): 18/06/2021

\begin{abstract}
RESUMEN
En las últimas décadas los compuestos tipo sándwich han tomado una mayor participación en la industria de la construcción, debido a su bajo costo y propiedades que caracterizan a estos compuestos. Entre las propiedades más importantes de estos resalta la conductividad térmica que se vincula directamente con el confort térmico en una edificación. En la actualidad, el Estado Peruano viene desarrollando proyectos en la lucha contra las heladas y el friaje, donde una de las principales intervenciones se enfoca en la construcción de viviendas sostenibles que brinden protección al poblador alto andino ante las inclemencias del clima. En ese sentido es necesario contar con materiales de construcción apropiados para las condiciones climáticas del lugar, y es ahí donde radica la importancia de esta investigación. En este trabajo se propone el estudio de la conductividad térmica a condiciones de temperatura cercanas a $0{ }^{\circ} \mathrm{C}$ de compuestos tipo sándwich formados a partir de materiales monolíticos más usados en construcción como el drywall, ladrillo, poliestireno expandido, lana de vidrio y lana poliéster. El estudio fue realizado mediante el empleo de un equipo de placa caliente con guarda de doble muestra, construido con los lineamientos establecidos por la norma ASTM C177. Las medidas realizadas de los compuestos tipos sándwich drywallpoliestireno expandido-drywall, drywall-lana de vidrio-drywall, drywall-lana poliéster-drywall, ladrillo-poliestireno expandidoladrillo, ladrillo-lana de vidrio-ladrillo y ladrillo-lana poliéster-ladrillo, dieron los siguientes resultados $0,0953 \mathrm{~W}^{\mathrm{W}} \mathrm{m}^{-1} .{ }^{\circ} \mathrm{C}^{-1}, 0,0675$ $\mathrm{W} \cdot \mathrm{m}^{-1} \cdot{ }^{\circ} \mathrm{C}^{-1}, 0,0833 \mathrm{~W} \cdot \mathrm{m}^{-1} \cdot{ }^{\circ} \mathrm{C}^{-1}, 0,0891 \mathrm{~W} \cdot \mathrm{m}^{-1} .{ }^{\circ} \mathrm{C}^{-1}, 0,0602 \mathrm{~W} \cdot \mathrm{m}^{-1} .{ }^{\circ} \mathrm{C}^{-1} \mathrm{y} 0,0709 \mathrm{~W} \cdot \mathrm{m}^{-1} .^{\circ} \mathrm{C}^{-1}$, respectivamente.
\end{abstract}

Palabras Clave: Compuesto tipo sándwich, Conductividad térmica, Confort térmico, Heladas, Viviendas sostenibles.

\section{ABSTRACT}

In recent decades sandwich composites have taken a greater share in the construction industry, due to their low cost and properties that characterize these composites. Among the most important properties of these, the thermal conductivity stands out, which is directly linked to the individual's thermal comfort in a building. Currently, the Peruvian State has been developing projects in the fight against frost and cold, where one of the main interventions focuses on the construction of sustainable homes that can help the high Andean population cope with the inclement cold. In this sense, it is necessary to have construction materials appropriate for the climatic conditions of the place, and that is where lies the importance of this research. This paper proposes the study of thermal conductivity at temperature conditions close to $0{ }^{\circ} \mathrm{C}$ of sandwich composites formed from monolithic materials most used in construction such as drywall, brick, expanded polystyrene, glass wool and polyester wool. The study was carried out through the use of a guarded hot plate equipment of double sample, built with the guidelines established by the ASTM C177 standard. The measurements made of the composites drywall-expanded polystyrene-drywall, drywall-glass wool-drywall, drywall-polyester wool-drywall, brick-expanded polystyrene-brick, brick-glass wool-brick, brick-polyester wool-brick, gave the following results $0.0953 \mathrm{Wm}^{-1} .{ }^{\circ} \mathrm{C}^{-1}, 0.0675 \mathrm{Wm}^{-1} .^{\circ} \mathrm{C}^{-1}, 0.0833 \mathrm{Wm}^{-1} .^{\circ} \mathrm{C}^{-1}, 0.0891$ $\mathrm{Wm}^{-1} .{ }^{\circ} \mathrm{C}^{-1}, 0.0602 \mathrm{Wm}^{-1} .{ }^{\circ} \mathrm{C}^{-1}, 0.0709 \mathrm{Wm}^{-1} .{ }^{\circ} \mathrm{C}^{-1}$ respectively.

Keywords: Sandwich compound, Thermal conductivity, Thermal comfort, Frost, sustainable homes.

1 Corresponding author:

E-mail: marck.182mr@gmail.com 


\section{INTRODUCCIÓN}

La actual revolución en la construcción ha permitido el desarrollo de nuevos material o compuestos que satisfagan las necesidades de las edificaciones. Entre estas resalta el aislamiento térmico, que esta principalmente relacionada a la demanda energética y al confort térmico. Esta necesidad del aislamiento cobra aun mayor importancia en los lugares donde la temperatura se encuentra por debajo de los $0^{\circ} \mathrm{C}$, como en las regiones altoandinas del Perú que año tras año son afectadas por las heladas, por ejemplo, en Puno en lo que va del año 2020 hasta la semana 26 se ha registrado la muerte de 2 niños menor de cinco años y 15 adultos mayores a causa de las infecciones respiratorias agudas $y$ neumonías por el descenso de temperatura que llegan hasta los $-18{ }^{\circ} \mathrm{C}[1]$. Bajo estas condiciones el calor en el interior de las viviendas se transfiere en gran cantidad al exterior de la misma, provocando un descenso abrupto de la temperatura. Es en ese sentido que, los materiales utilizados para la construcción de viviendas en dichas zonas deben cumplir con determinadas características y propiedades que puedan catalogarlas como adecuadas para ser habitadas. Entre estas podemos considerar el valor de la conductividad térmica, parámetro que da a conocer la facilidad con la que un material permite el flujo de calor a través de sí mismo, como un indicador del aislamiento térmico que pueda brindar un determinado material. En el presente estudio se evaluó la conductividad térmica de compuestos tipo sándwich que fueron elaborados a partir de materiales usados convencionalmente en construcción. Estos compuestos se constituyeron de la siguiente manera: drywall-poliestireno expandidodrywall, drywall-lana de vidrio-drywall, drywall-lana poliéster-drywall, ladrillo-poliestireno expandidoladrillo, ladrillo-lana de vidrio-ladrillo, y ladrillo-lana poliéster-ladrillo. Este trabajo se ha realizado mediante el empleo de un equipo de placa caliente con guarda de doble muestra, denominado LAMBDA UNI, que ha sido construido bajo los lineamientos de la norma ASTM C177 [2], y se encuentra ubicado en el Laboratorio de Ingeniería Física de la Facultad de Ciencias de la Universidad Nacional de Ingeniería (UNI). Por medio de este equipo se puede determinar el valor de la conductividad térmica como se indica en la ecuación (1) [3].

$$
\lambda=\frac{\dot{Q} L}{A\left(T_{1}-T_{2}\right)}
$$

Donde, $\lambda$ es la conductividad térmica $\left(\mathrm{W} \cdot \mathrm{m}^{-1} \cdot{ }^{\circ} \mathrm{C}^{-1}\right), \dot{Q}$ el flujo de calor a través del material (W), Aes el área de medición $\left(\mathrm{m}^{2}\right)$, Lrepresenta el espesor de la material (m), $T_{1}$ y $T_{2}$ son las temperaturas caliente y fría respectivamente en las superficies opuestas del material $\left({ }^{\circ} \mathrm{C}\right)$, perpendiculares al flujo de calor.

\section{ANTECEDENTES}

En el Perú, el acondicionamiento térmico en las viviendas es un tema que cada año va tomando mayor importancia sobre todo en las regiones que son afectados por las heladas y el friaje, en este sentido el Estado Peruano viene impulsando y mejorando con cada proyecto la implementación de viviendas acondicionadas térmicamente para la mejora de la calidad de vida del poblador altoandino, he ahí donde se viene promoviendo a su vez la investigación de nuevas alternativas en materia de construcción.

En el 2014, en la Universidad de San Cristóbal de Huamanga se realizaron mediciones de conductividad térmica de materiales usados en edificaciones de la región de Ayacucho mediante una la elaboración de un medidor de conductividad térmica (MTC) usando el método de placa caliente con guarda basado en la norma ASTM C177 [4]. Una de las muestras evaluadas fue madera tornillo, el estudio consistió en realizar las medidas a distintos espesores de la muestra obteniendo como resultado una conductividad promedio de $0,141 \mathrm{~W} \cdot \mathrm{m}^{-1} \cdot{ }^{\circ} \mathrm{C}^{-1} \pm 0,02 \mathrm{~W} \cdot \mathrm{m}^{-1} \cdot{ }^{\circ} \mathrm{C}^{-1}$ para un rango de espesores de $0,56 \mathrm{~cm}$ a $3,14 \mathrm{~cm}$ [5].

En el 2015, la Universidad de Ingeniería y Tecnología presentó los resultados de las mediciones de conductividad térmica realizadas a paneles de Stipa ichu mediante un sistema de medición de conductividad térmica unidireccional diseñado y fabricado en base a la norma ASTM C177. Los resultados obtenidos muestran que la conductividad térmica del ichu varia de $0,047 \mathrm{~W} \cdot \mathrm{m}^{-1} .{ }^{\circ} \mathrm{C}^{-1}$ a $0,113 \mathrm{~W} \cdot \mathrm{m}^{-}$ ${ }^{1} .{ }^{\circ} \mathrm{C}^{-1}$ dependiendo de su densidad principalmente [6].

En el 2020, en un artículo presentado en la IOPS se mostraron los resultados de un estudio de las propiedades térmicas del adobe con y sin Stipa ichu como compósito; para este fin se empleó un aparato de placa caliente con guarda (CNM-PNE-16) ubicado en el laboratorio de CENAM México, la data obtenida y su posterior análisis demostró la ventaja de este material como aislante térmico frente a otros materiales de construcción convencionales [7].

\section{METOdOLOGÍA}

La determinación de la conductividad térmica de los materiales compuestos tipo sándwich elaborados en el presente estudio, se realizó mediante el empleo de un equipo de placa caliente con guarda de doble muestra. Este equipo es denominado LAMBDA UNI y fue elaborado bajo los lineamientos de la Norma ASTM 
- C177, en la cual también se establecen los criterios básicos para una correcta medición.

La realización del presente estudio consistió en la elaboración de muestras de compuestos tipo sándwich, y un asilamiento de borde hecho de poliestireno extrudido. Para después ser introducido en el equipo LAMBDA UNI y realizar la medición de los parámetros que determinan el valor de la conductividad térmica del compuesto.

\subsection{MATERIALES COMPUESTOS}

Se conoce como material compuesto a cualquier material multifase que presente una proporción significativa entre sus componentes, y se constituyan de modo que se obtenga una mejor combinación de propiedades [8]. Estos materiales compuestos se clasifican en base a las propiedades de sus fases constituyentes, de sus propiedades relativas $y$ geometrías correspondientes, en la Fig. 1 se muestra un esquema de esta clasificación.

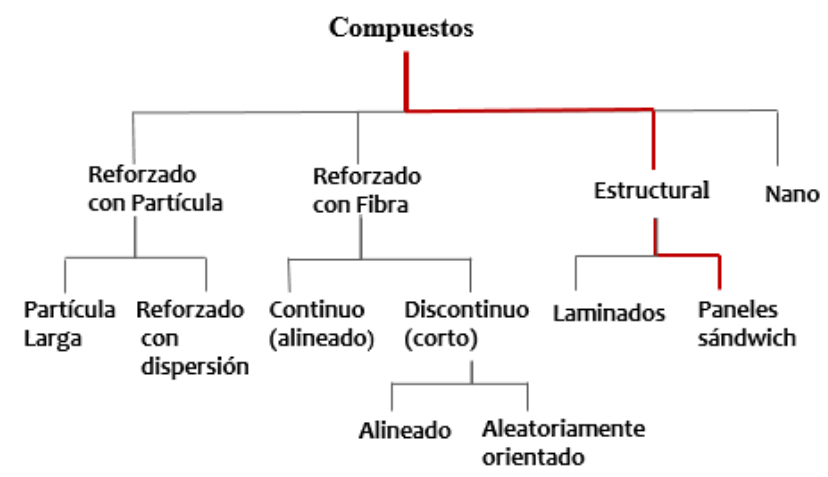

Fig. 1. Clasificación de materiales compuestos [8].

De acuerdo a la Fig. 1, los materiales compuestos se clasifican en 4 tipos. De estos los compuestos estructurales de tipo paneles sándwich, más conocidos como compuestos tipo sándwich, son los que se abordan en el presente estudio.

Los compuestos estructurales tipo sándwich constituidos por dos hojas externas, llamadas también caras o capas, y unidas de forma adhesiva a un núcleo más grueso, tal como se muestra en la Fig. 2. Las capas exteriores son hechas por lo general de un material rígido y resistente. El material del núcleo suele ser liviano, con una densidad menor a $0,25 \mathrm{~g} / \mathrm{cm}^{3}$. La rigidez del compuesto depende principalmente de las propiedades del material y espesor del núcleo [8].

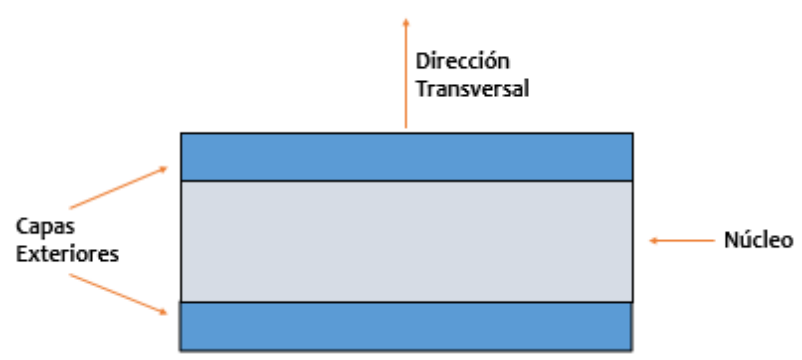

Fig. 2. Diagrama esquemático de un compuesto estructural tipo sándwich [8].

Un beneficio del compuesto es principalmente su costo, ya que el núcleo suele ser de mayor espesor y es el de menor valor económico. Otros beneficios relacionados a sus características físicas se mencionan a continuación:

a Ligereza. Gracias a la baja densidad y mayor espesor del núcleo en comparación al de las capas, el compuesto reduce en gran medida su densidad.

b Rigidez a flexión. En la Tabla I se muestran tres arreglos de compuestos, el primero solo formado por dos capas del mismo material y de igual espesor $n / 2$, el segundo formado por dos capas de espesor $\mathrm{n} / 2$ y un núcleo de espesor $\mathrm{n}$, y el tercero formado por dos capas de espesor $\mathrm{n} / 2$ y un núcleo de espesor $3 \mathrm{n}$.

Tabla I

Rigidez a flexión y resistencia relativa de un compuesto estructural tipo sándwich [9].

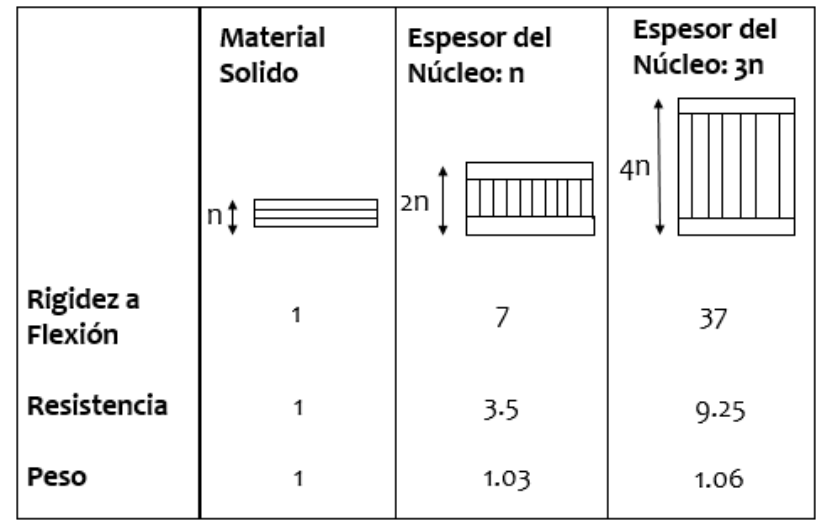

De la Tabla I se observa que, con el aumento del espesor en el núcleo, la rigidez a flexión se incrementa en la segunda y tercera configuración respecto a la primera, de igual modo la resistencia también se incrementa considerablemente. El peso en cambio no sufre un incremento notorio, con lo que el incremento en su volumen conlleva a una disminución en su densidad. 
c Aislamiento térmico. Los materiales usados para el núcleo son por lo general materiales aislantes, y al ser este de mayor espesor, se consigue que el compuesto también actué como aislante [10].

\subsection{CONDUCTIVIDAD TÉRMICA}

A principios del siglo XIX. El matemático y físico Fourier estableció la teoría matemática de la conducción de calor en base a sus resultados experimentales. La Fig. 3 muestra un esquema del experimento realizado por Fourier.

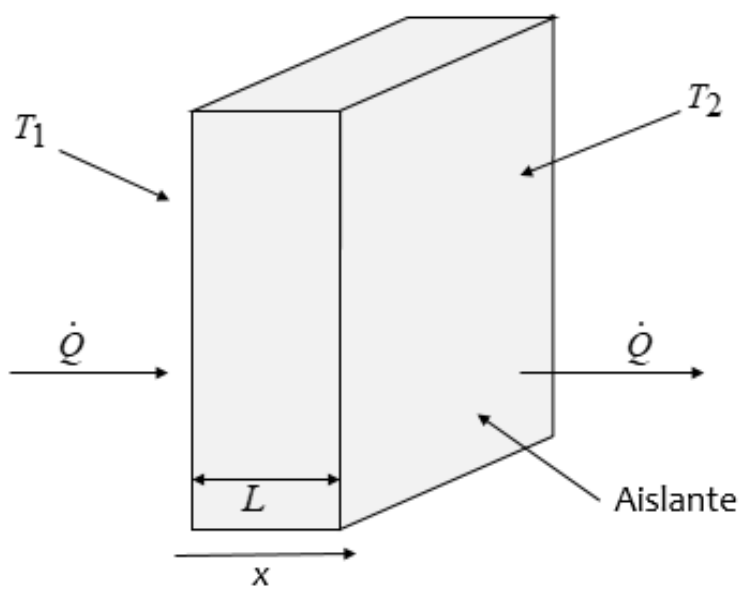

Fig. 3. Conducción de calor unidimensional en un sólido [11].

En la Fig. 3, la superficie del lado izquierdo del sólido rectangular se mantiene a una temperatura $T_{1}$ mientras la superficie del lado derecho se mantiene a una temperatura $T_{2}$, las demás superficies del sólido se aíslan de modo que se consiga un flujo de calor unidireccional en la dirección $X$. Bajo estas condiciones se determinó que la razón de transferencia de calor $\dot{Q}$

Tabla II

Conductividad térmica de algunos materiales de construcción [14]-[18].

\begin{tabular}{|l|c|}
\hline \multicolumn{1}{|c|}{ Materiales } & Conductividad térmica W.m ${ }^{-1} .{ }^{\circ} \mathrm{C}^{-1}$ \\
\hline Poliestireno Expandido (Tecnopor) & $0,0316-0,0354$ \\
Panel de Lana de Vidrio & 0,0407 \\
Lana Poliéster Ecoterm & 0,063 \\
Plancha Drywall Volcanita 1/2" (yeso y cartón) & 0,26 \\
Ladrillo Aislante & 0,15 \\
Ladrillo Rojo & 0,6 \\
\hline
\end{tabular}

Los valores de conductividad térmica de los materiales descritos en la Tabla II, son valores referenciales que han sido obtenidos bajo determinadas condiciones de medida. es directamente proporcional al área de sección transversal $A$ (a través de la cual fluye el calor), a la diferencia de temperatura $\left(T_{1}-T_{2}\right)$, e inversamente proporcional al espesor $L$ del material [11]. Como se muestra en la ecuación (2).

$$
\dot{Q} \alpha \frac{A\left(T_{1}-T_{2}\right)}{L}
$$

Escribiendo esta relación como una igualdad obtenemos la ecuación (3):

$$
\dot{Q}=\frac{\lambda A\left(T_{1}-T_{2}\right)}{L}
$$

Donde $\lambda$, es la constante de proporcionalidad llamada conductividad térmica, y se define como la razón de transferencia de calor a través de un espesor unitario del material por unidad de área por unidad de diferencia de temperatura. La conductividad térmica de un material es una medida de la capacidad del material para conducir calor. Un valor elevado para la conductividad térmica indica que el material es un buen conductor del calor y un valor bajo indica que es un mal conductor o que es un aislante [12]. La conductividad térmica de un material depende de la composición química de la sustancia o sustancias que lo componen, de la fase (gaseosa, líquida o sólida) en que se encuentra, de su estructura cristalina si se trata de un sólido, de la temperatura, la humedad y presión a que está sometido y de sí es o no un material homogéneo [13]. En la Tabla II se dan las conductividades térmicas de algunos materiales más usados en construcción.

\subsection{EQUIPO DE MEDICIÓN DE CONDUCTIVIDAD TÉRMICA LAMBDA UNI}

El equipo medidor de conductividad térmica LAMBDA UNI fue diseñado y construido en base a la norma ASTM C-177, en la Fig. 4 se muestra un esquema del diseño equipo. 


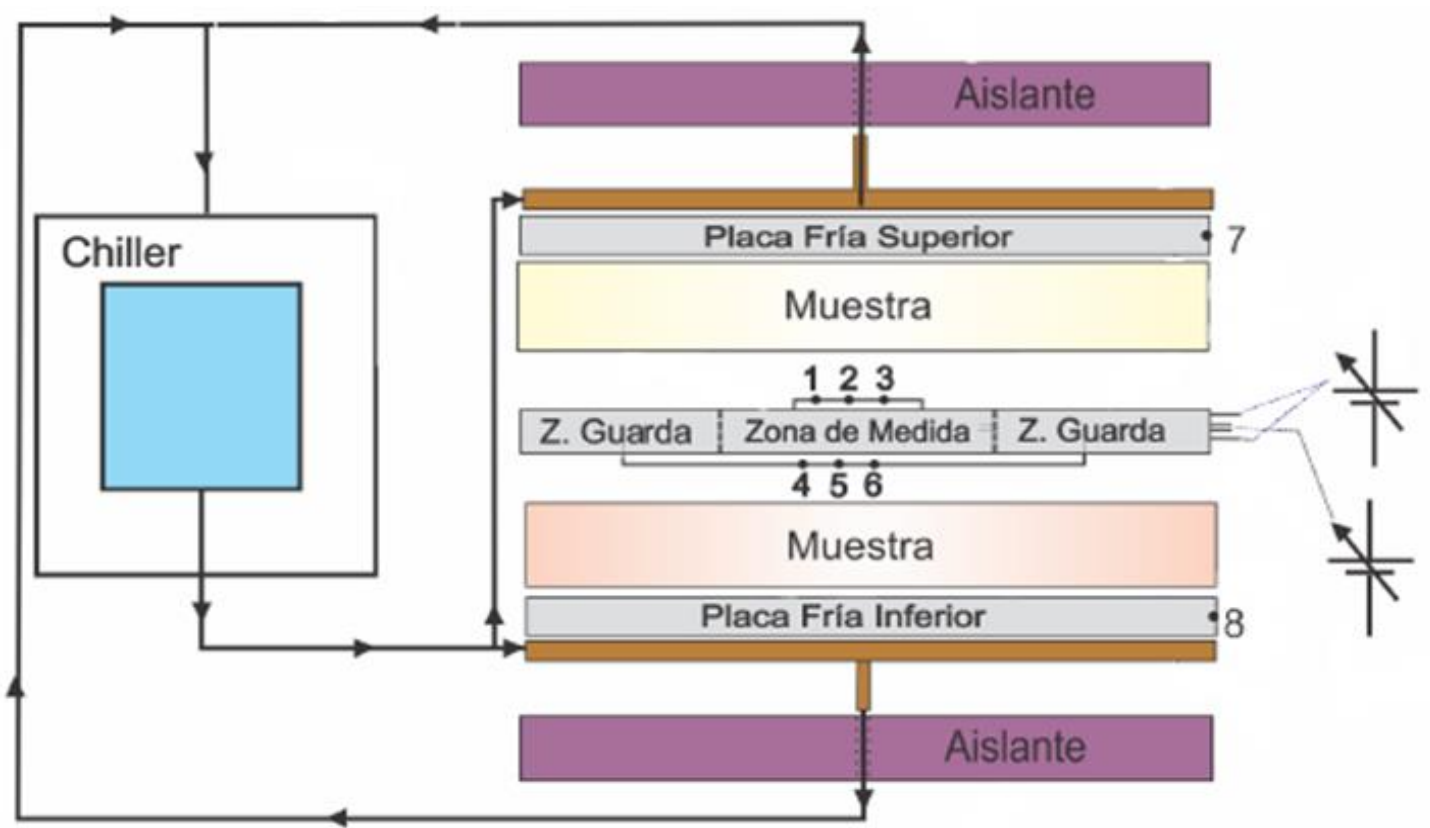

Fig. 4. Esquema del equipo de placa caliente con guarda LAMBDA UNI. Los números de indican las posiciones de los termopares [19].

Este esquema es correspondiente a un equipo de placa caliente con guarda de doble muestra y flujo de calor unidimensional. En esta configuración se realizan ensayos de dos muestras del mismo tipo y en igualdad de condiciones, idealmente se genera un flujo de calor vertical desde la zona de medición hacia las placas frías

A continuación, se da una breve descripción de los componentes del equipo de placa caliente con guarda LAMBDA UNI:

Conjunto de superficies frías: son dos placas frías, una ubicada en la parte superior de una de las muestras y la otra ubicada en la parte inferior de la otra muestra. Su función es disipar el calor generado en el conjunto de placa caliente y mantener a una temperatura homogénea a las superficies de la muestra que están en contacto con estos. Estas placas se encuentran conectadas a un sistema de refrigeración, un chiller, que permite fijar su temperatura a un valor deseado.

Conjunto de superficies calientes: son dos placas calientes, una ubicada en la zona de medición, y que se encuentra aislada térmicamente por un gap de la otra placa concéntrica, zona de guarda. Estas cumplen un rol importante de acuerdo a su ubicación.

La placa en la zona de medición: tiene como función proporcionar la potencia (flujo de calor por unidad de tiempo) para la medición mediante un calentador intercalado entre sus caras. El tamaño de la zona de medición define el volumen real de la prueba.

La placa en la zona de guarda: tiene como función brindar las condiciones térmicas apropiadas dentro del volumen de prueba con el fin de reducir el flujo lateral de calor dentro del equipo y está ubicado concéntrica y alrededor de la placa caliente de la zona de medición. Se compone de uno o más calentadores intercalados entre sus caras.

Las muestras: deben cumplir con las recomendaciones establecidas en la Norma ASTM C177. Entre ellas, el espesor máximo que debe ser un tercio de la dimensión lineal máxima de la zona de medición, las dimensiones de la muestra deben ser tal que cubran toda la zona de medición (siendo deseable que incluya el gap y el área de la zona de guarda). Ambas muestras deben ser tan similares en espesor y características térmicas como sea posible. De igual modo, si lo requieren, las muestras deben contar con un aislamiento que limite el flujo de calor en su borde lateral.

\subsection{ECUACIONES PARA EL CÁLCULO DE LA CONDUCTIVIDAD TÉRMICA}

En un equipo de placa caliente con guarda ideal y de doble muestra, cuando se tienen dos muestras homogéneas e isotrópicas, del mismo material y dimensiones, además, considerando que las superficies isotérmicas frías y calientes presentan contacto perfecto con las muestras, entonces el flujo de calor a través de las muestras es unidimensional (flujo de calor unidireccional) e independiente del tiempo (estado estacionario). Bajo estas condiciones la conductividad térmica $\lambda$ de las muestras se puede determinar a partir del flujo de calor $\dot{\boldsymbol{Q}}$, que atraviesa el área total de medición, $\mathbf{2 A}$; el gradiente de temperaturas entre la zona de medición y el promedio de temperaturas de las placas frías $\boldsymbol{\Delta T} ;$ y el 
espesor promedio de las muestras $\boldsymbol{e}$, en base a la Ley de Fourier mediante la ecuación (4) [3].

$$
\lambda=\frac{\dot{Q} e}{2 A(\Delta T)}
$$

Los equipos de placa caliente con guarda como cualquier otro equipo de medición están propensos errores en su medición. Para un equipo de placa caliente con guarda, los errores en las mediciones son la incertidumbre en: el flujo de calor, la diferencia de temperaturas, el área de medición, y el espesor de la muestra. De este modo la incertidumbre se determinó mediante la ecuación (5) de propagación de errores como se muestra a continuación [2]:

$$
\frac{\mu_{\lambda}}{\lambda}=\sqrt{\left(\frac{\mu_{\dot{Q}}}{\dot{Q}}\right)^{2}+\left(\frac{\mu_{e}}{e}\right)^{2}+\left(\frac{\mu_{2 A}}{2 A}\right)^{2}+\left(\frac{\mu_{\Delta T}}{\Delta T}\right)^{2}}
$$

Donde, $\boldsymbol{\mu}_{\lambda}$ es la incertidumbre en el valor de la conductividad térmica, $\boldsymbol{\mu}_{\boldsymbol{Q}}$ es la incertidumbre del flujo de calor $(\mathrm{W}), \boldsymbol{\mu}_{\boldsymbol{e}}$ es la incertidumbre del espesor (m), $\boldsymbol{\mu}_{A}$ es la incertidumbre del área total de medición $\left(\mathrm{m}^{2}\right)$, $\boldsymbol{\mu}_{\Delta T}$ es la incertidumbre de la diferencia de temperatura entre la placa caliente y la placa fría $\left({ }^{\circ} \mathrm{C}\right)$.

Además, el valor del flujo de calor resulta del producto de la corriente y el voltaje suministrado de la fuente a la placa caliente. Su incertidumbre queda determinada por la ecuación (6).
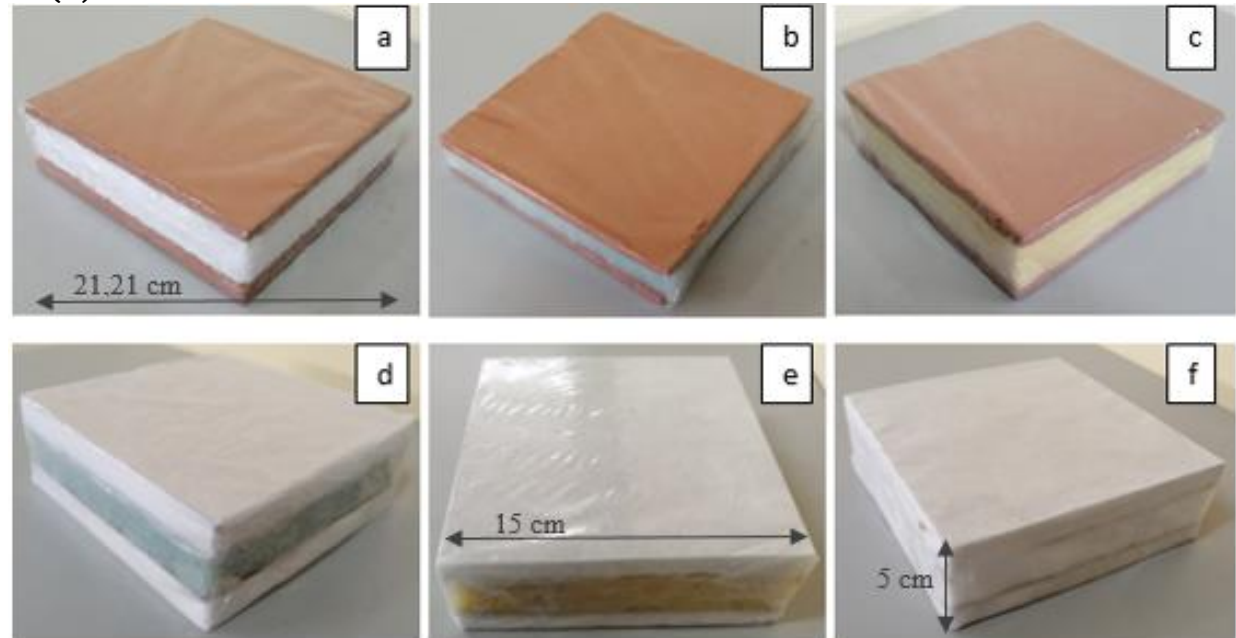

Fig. 5. Muestras tipo sándwich: (a) Ladrillo-poliestireno expandido-ladrillo (LP-PLEX-LP), (b) Ladrillo-lana poliéster-ladrillo (LP-ECOTERM-LP), (c) Ladrillo-lana de vidrio-ladrillo (LP-LV-LP), (d) Drywall-lana poliéster-drywall (DW-ECOTERM-DW), (e) Drywall-lana de vidrio-drywall (DRY-LVDRY), (f) Drywall-poliestireno expandido-drywall (DRY-PLEX-DRY).

Como se puede observar estos compuestos tienen una forma paralelepípedo, de base cuadrada de $15 \mathrm{~cm}$ de lado y una altura que varía de acuerdo al espesor de sus materiales constituyentes, esta altura máxima no debe superar los $5 \mathrm{~cm}$ por recomendación de la Norma ASTM C177. 
Dada las dimensiones de la muestra ha sido necesario la elaboración de aislantes de borde para cada una de ellas. En la Fig. 6 se muestra el aislante de borde de la muestra de ladrillo-lana de vidrio-ladrillo.
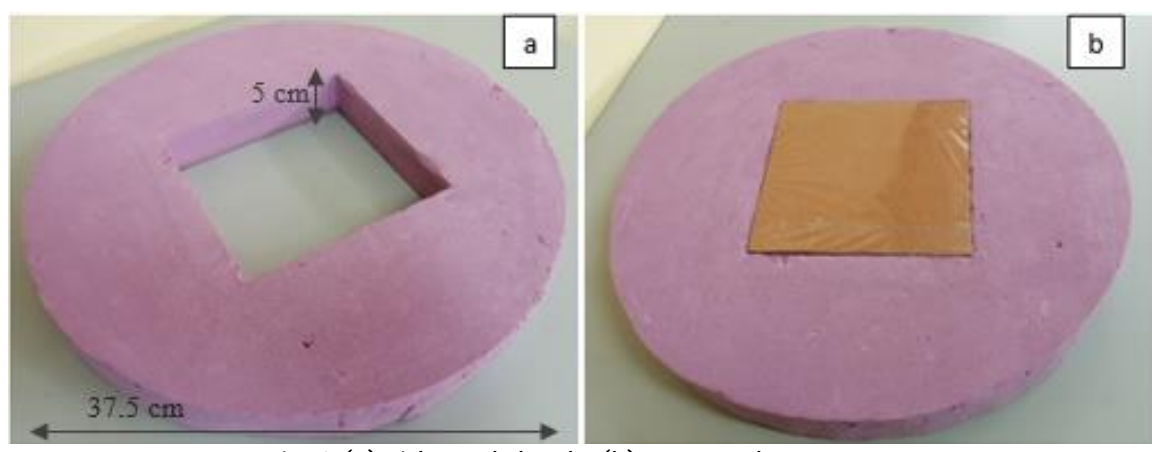

Fig. 6. (a) Aislante de borde, (b) Muestra de LP-LV-LP .

\section{ANÁLISIS DE RESULTADOS}

El valor de la conductividad térmica determinado en cada ensayo, proviene de una recopilación y análisis de datos obtenidos del equipo LAMBDA UNI. Estos datos son exportados a una hoja de cálculo, donde son sintetizados para su a análisis gráfico en Origin. Una vez determinado el rango total donde se evaluó el valor de la conductividad térmica, donde la diferencia de temperaturas entre la zona de medición y la zona de guarda no difieran en más de $0,2{ }^{\circ} \mathrm{C}$ en el estado estacionario durante al menos 1 hora y media, se procede con el cálculo correspondiente de acuerdo a la ecuación (4).

\subsection{DATOS GRÁFICOS}

Los ensayos pueden durar de uno a dos días en una primera prueba a fin de asegurar el estado estacionario para determinadas condiciones de temperaturas en las que se requieran realizar dicho ensayo. A continuación, en la Fig. 7 se muestra el comportamiento de las de temperaturas y las potencias suministradas en los componentes del equipo LAMBDA UNI, para el ensayo del compuesto LP-LV-LP.

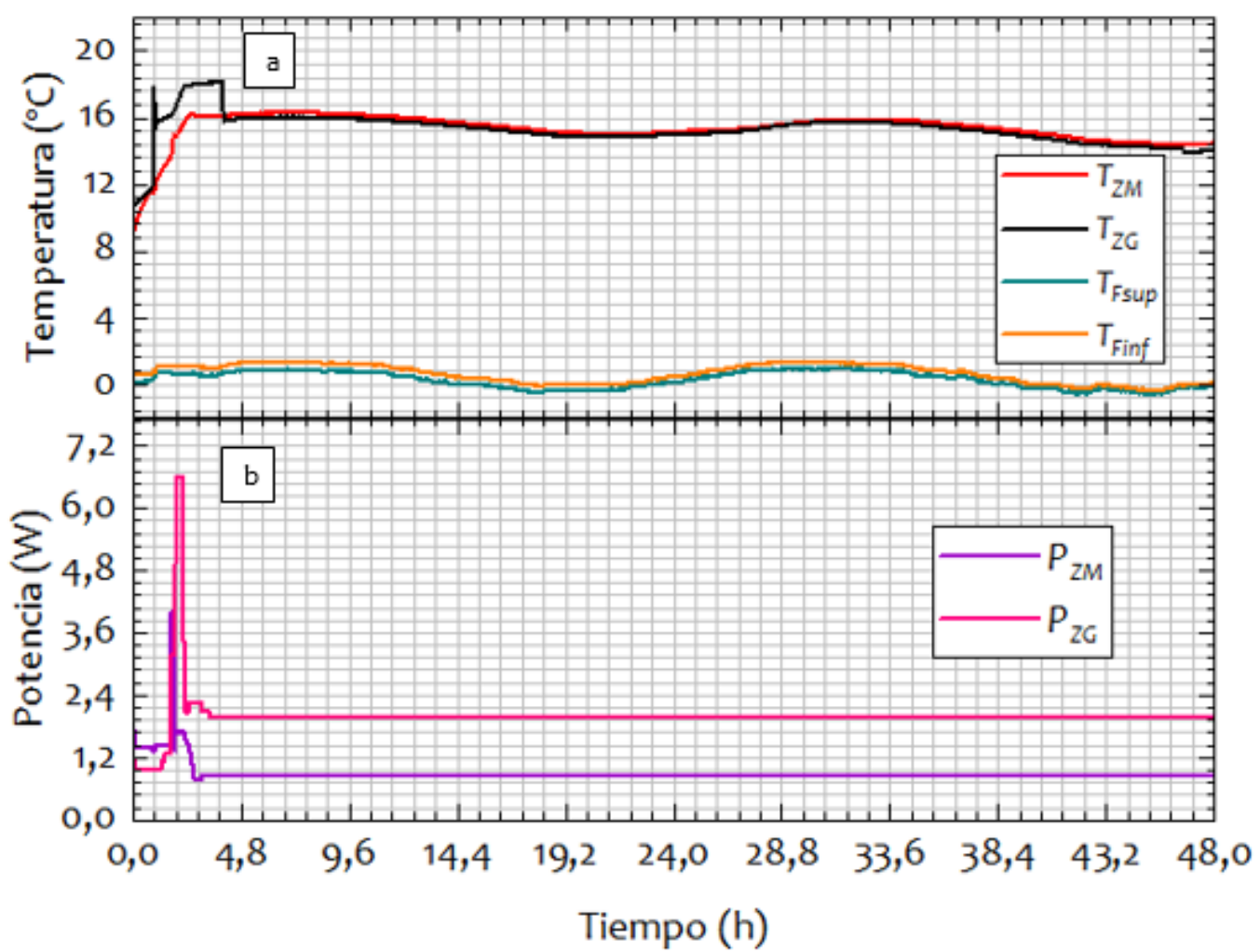

Fig. 7. Comportamiento en el tiempo para el ensayo de conductividad térmica del compuesto LP-LV-LP (a) muestra el comportamiento térmico de las placas frías y la placa caliente y (b) muestra las variaciones realizadas a las potencias suministradas a la zona de guarda $\left(P_{\mathrm{ZC}}\right)$ y zona de medición $\left(P_{Z M}\right)$. 
De acuerdo a los valores de temperatura observados en la Fig. 7 y al tratamiento de datos realizado, se consideró para la evaluación de la conductividad térmica los datos encapsulados desde las 28,87 horas hasta las 30,37 horas. En la Fig. 8 se observa el comportamiento térmico del valor absoluto de la diferencia de temperatura entre la zona de guarda y zona de medición en el rango de tiempo seleccionado.

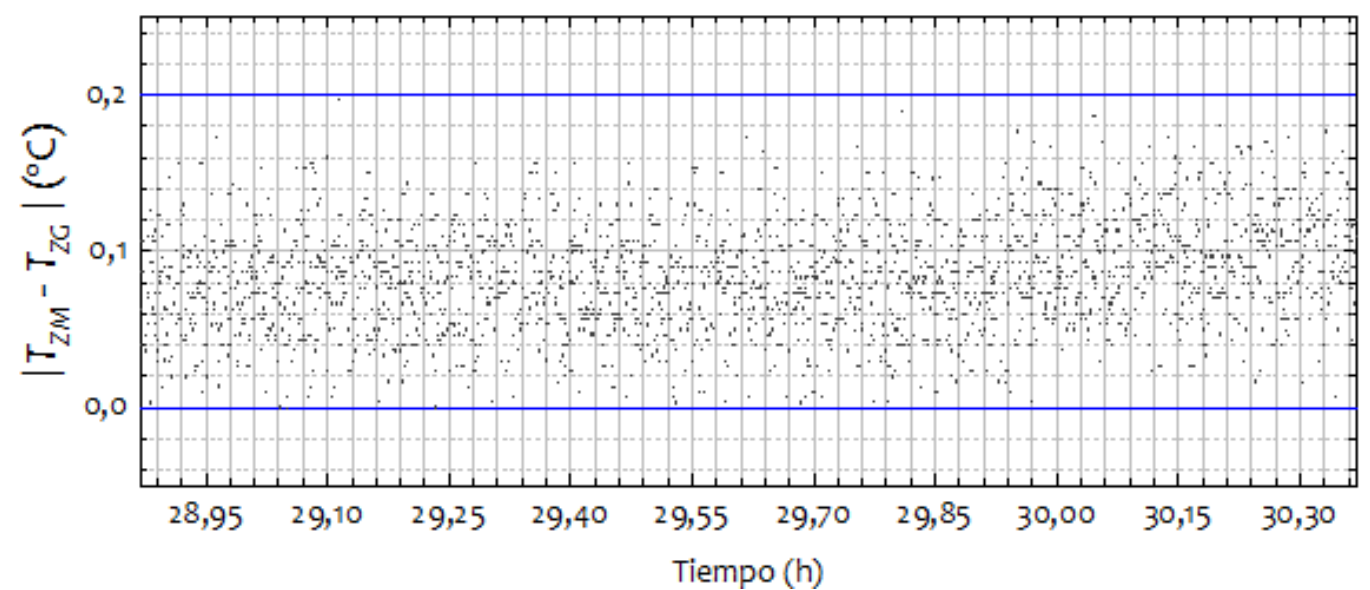

Fig. 8. Comportamiento térmico del valor absoluto de la diferencia de temperatura entre la zona de guarda y zona de medición entre las 28.87 horas hasta las 30,37 horas de trascurrida la medición.

De la Fig. 8 la diferencia de temperatura entre la zona de medición y zona de guarda es menor a $0,2{ }^{\circ} \mathrm{C}$, por lo que se cumple el criterio establecido en la Norma ASTM-C177.

De modo similar se realizó el análisis de datos para los compuestos de LP-PLEX-LP, LP-ECOTERM-LP, LP-LVLP, DW-ECOTERM-DW, DW-LV-DW, y DRY-PLEX-DW.

\subsection{DATOS EXPERIMENTALES}

Después de considerar la información gráfica y realizar el análisis de datos correspondientes a cada ensayo, y considerando un área total de medición fija $\mathbf{2 A}=0,03590$ en la Tabla III se presentan los valores encontrados para cada parámetro en el cálculo de la conductividad térmica, así como el valor de la conductividad térmica y su correspondiente incertidumbre.

TABLA ॥

Datos y Resultados de conductividad térmica en cada ensayo

\begin{tabular}{|l|ccccc|}
\hline MUESTRA & $\dot{Q}(\mathrm{~W})$ & $e(\mathrm{~m})$ & $\Delta T\left({ }^{\circ} \mathrm{C}\right)$ & $\lambda\left(\mathrm{W} \cdot \mathrm{m}^{-1} \cdot{ }^{\circ} \mathrm{C}^{-1}\right)$ & $U_{\lambda}\left(\mathrm{W} \cdot \mathrm{m}^{-1} \cdot{ }^{\circ} \mathrm{C}^{-1}\right)$ \\
\hline (a) LP-PLEX-LP & 1,14 & 0,04116 & 14,67 & 0,0891 & 0,0102 \\
(b) LP-ECOTERM-LP & 1,08 & 0,03648 & 15,48 & 0,0709 & 0,0077 \\
(c) LP-PLV-LP & 0,865 & 0,03648 & 14,60 & 0,0602 & 0,0069 \\
(d) DW-ECOTERM-DW & 0,983 & 0,04528 & 14,88 & 0,0833 & 0,0094 \\
(e) DW-PLV-DW & 0,743 & 0,04841 & 14,85 & 0,0675 & 0,0076 \\
(f) DW-PLEX-DW & 1,00 & 0,05034 & 14,72 & 0,0953 & 0,0107 \\
\hline
\end{tabular}

\section{CONCLUSIONES}

Se logró determinar el valor de conductividad térmica de los compuestos LP-PLEX-LP, LP-ECOTERM-
LP, LP-LV-LP, siendo el compuesto con menor conductividad térmica el que tiene como núcleo a la lana de vidrio con un valor de $0,0602 \mathrm{~W} \cdot \mathrm{m}^{-1} \cdot{ }^{\circ} \mathrm{C}^{-1} \pm$ $0,0069 \mathrm{~W} \cdot \mathrm{m}^{-1} \cdot{ }^{\circ} \mathrm{C}^{-1}$, seguido del compuesto con el núcleo 
lana poliéster con un valor de $0,0709 \mathrm{~W} \cdot \mathrm{m}^{-1} \cdot .^{\circ} \mathrm{C}^{-1} \pm$ $0,0077 \mathrm{~W} \cdot \mathrm{m}^{-1} \cdot{ }^{\circ} \mathrm{C}^{-1}$, y finalmente el compuesto con núcleo de poliestireno expandido con un valor de $0,0891 \mathrm{~W} \cdot \mathrm{m}^{-1} \cdot{ }^{\circ} \mathrm{C}^{-1} \pm 0,0102 \mathrm{~W} \cdot \mathrm{m}^{-1} \cdot{ }^{\circ} \mathrm{C}^{-1}$.

De igual manera se logró determinar el valor de conductividad térmica de los compuestos DWECOTERM-DW, DW-LV-DW, y DRY-PLEX-DW, siendo el compuesto con menor conductividad térmica el que tiene como núcleo a la lana de vidrio con un valor de $0,0675 \mathrm{~W} \cdot \mathrm{m}^{-1} \cdot{ }^{\circ} \mathrm{C}^{-1} \pm 0,0076 \mathrm{~W} \cdot \mathrm{m}^{-1} \cdot{ }^{\circ} \mathrm{C}^{-1}$, seguido del compuesto con el núcleo lana poliéster con un valor de $0,0833 \mathrm{~W} \cdot \mathrm{m}^{-1} \cdot{ }^{\circ} \mathrm{C}^{-1} \pm 0,0094 \mathrm{~W} \cdot \mathrm{m}^{-1} \cdot{ }^{\circ} \mathrm{C}^{-1}, \mathrm{y}$ finalmente el compuesto con núcleo de poliestireno expandido con un valor de $0,0953 \mathrm{~W} \cdot \mathrm{m}^{-1} .{ }^{\circ} \mathrm{C}^{-1} \pm 0,0107 \mathrm{~W} \cdot \mathrm{m}^{-1} .{ }^{\circ} \mathrm{C}^{-1}$.

El valor de la incertidumbre para la conductividad térmica de cada compuesto tiene como factores porcentuales $6,46 \%$ debido a la potencia suministrada, 4,02 \% debido al espesor, 0,86 \% debido al área de medición y 88,66 \% debido al gradiente de temperatura.

Los resultados encontrados para cada compuesto son menores de $0,1 \mathrm{~W} \cdot \mathrm{m}^{-1} \cdot{ }^{\circ} \mathrm{C}^{-1} \mathrm{e}$ incertidumbre menor al $11,5 \%$ del valor de la conductividad térmica en cada ensayo. Estos valores bajos en conductividad térmica dan pase a la posibilidad de considerar estos compuestos tipo sándwich como alternativas viables para la construcción en las zonas afectadas por las heladas.

\section{AGRADECIMIENTOS}

Este trabajo se llevó a cabo bajo el auspicio del Consejo Nacional de Ciencia y Tecnología del Perú (CONCYTEC) bajo el convenio Nº24-2016-FONDECYT, el Vicerrectorado de Investigación de la Universidad Nacional de Ingeniería (VRI-UNI) bajo el proyecto MFFC-4-2019, y a la Facultad de Ciencias de la Universidad Nacional de Ingeniería por la financiación recibida por los proyectos formativa durante los años 2019 y 2020.

\section{REFERENCIAS}

[1] C. Fernandez, "Puno: neumonías por bajas temperaturas causan la muerte de 17 niños y ancianos," El Comercio, 2020. https://bit.ly/347mtmC (accessed Jul. 08, 2020).

[2] ASTM (2004). "ASTM C177: Standard Test Method for Steady-State Heat Flux Measurements and Thermal Transmission Properties by Means of the Guarded HotPlate Apparatus." Whashingtion D.C., [Online]. Available: https://www.astm.org/DATABASE.CART/HISTORICAL/C17704.htm.
Transmission Properties Under Steady-," Annu. B. ASTM Stand., vol. 14, no. June, pp. 1-13, 2012, doi: 10.1520/C104507R13.Copyright.

[4] K. Janampa Quispe, "Conductividad Térmica de Materiales Utilizados en Edificaciones de la Región de Ayacucho," 2014.

https://dina.concytec.gob.pe/appDirectorioCTI/VerDatosInv estigador.do?id_investigador $=17520$ (accessed May 24, 2020).

[5] K. Janampa, O. Cerón, O. Morales, y J. Oré, “Thermal characterization of materials used in rural housing constructions in Ayacucho, Peru," J. Phys. Conf. Ser., vol. 1433, no. 1, 2020, doi: 10.1088/1742-6596/1433/1/012004.

[6] J. Noel et al., "Assessment of Ichu Fibers as Non Expensive Thermal Insulation System for the Andean Regions," 2015, [Online]. Available: https://repositorio.utec.edu.pe/handle/UTEC/28?mode=full.

[7] J. M. Pinas, L. Lira, M. Horn, J. L. Solis, y M. M. Gómez, "Influence of Stipa ichu on the thermal and mechanical properties of adobe as a biocomposite material," J. Phys. Conf. Ser., vol. 1433, no. 1, 2020, doi: 10.1088/17426596/1433/1/012003.

[8] W. Callister y D. Rethwisch, Composites, 9th ed. United States of America, 2013.

[9] HexCel Composites, "Honeycomb sandwich design technology," HexWeb Honeycomb Sandw. Des. Technol., no. AGU 075b, pp. 1-28, 2000, [Online]. Available: https://www.hexcel.com/user_area/content_media/raw/Ho neycomb Sandwich_Design_Technology.pdf.

[10] J. M. Malavia Otero, "Caracterización de paneles sandwich hibridos frp con alma de nido de abeja de aluminio", Tesis de pregrado, Universidad Politécnica de Valencia, 2012.

[11] R. W. Serth and L. Thomas, "Heat Conduction," in Process Heat Transfer, 2nd ed., Texas: Academic Press, 2014, pp. 130.

[12] Y. A. Cengel and A. J. Ghajar, Transferencia de Calor y Masa, 4th ed. Mexico DF, 2011.

[13] A. J. Chapman, Transmision del Calor, 3rd ed. Madrid: MCMILLAN PUBLISHING COMPANY, 1990.

[14] A. L. P. Robert R. Zarr, "SRM 1453, Expanded Polystyrene Board, for Thermal Conductivity from $281 \mathrm{~K}$ to $313 \mathrm{~K}$," 2012. [En linea]. Disponible en: http://dx.doi.org/10.6028/NIST.SP. 260-175.

[15] AAISLACORP 2000, "PANEL DE LANA DE VIDRIO." 2018 [En linea]. Disponible en: https://www.aaislacorp.com/producto/panel-de-lana-devidrio/.

[16] Texeco (2006). "Aislante Térmico y Absorbente Acústico (Ecoterm)." p. 1, [En linea]. Disponible en : http://www.texecoperu.com.pe/assets/docs/FICHA_ECOTE RM.pdf.

[17] VOLCAN, "Planchas de Yeso Cartón - Volcanita ST." pp. 1-8, [Online]. Available: http://www.especificar.cl/fichas/planchas-de-yeso-cartonvolcanita-st.

[18] H. D. Young y R. A. Freedman, Temperatura y Calor, 12th ed. México.: Pearson, 2009.

[19] F. S. Espinoza Castillo, “Caracterización Térmica, Estructural y Mecánica de la Ignimbrita Aeropuerto de Arequipa", Tesis de pregrado, Universidad Nacional de Ingenieria, Lima, 2020. 